Alloyed surfaces: new substrates for graphene growth

C. Tresca, N.I. Verbitskiy, A. Fedorov, A. Grüneis, G. Profeta

PII:

S0039-6028(17)30469-7

DOI:

10.1016/j.susc.2017.08.002

Reference:

SUSC 21066

To appear in:

Surface Science

Surface Science

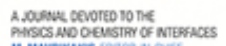

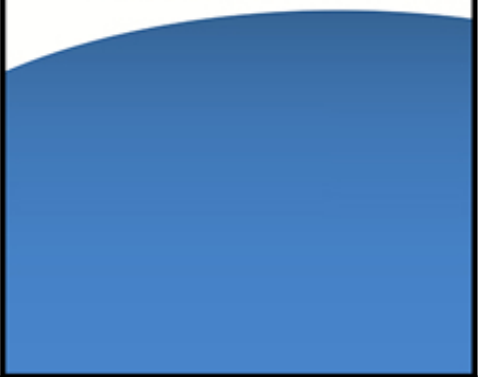

Received date:

27 June 2017

Revised date:

26 July 2017

Accepted date:

1 August 2017

Please cite this article as: C. Tresca, N.I. Verbitskiy, A. Fedorov, A. Grüneis, G. Profeta, Alloyed surfaces: new substrates for graphene growth, Surface Science (2017), doi: 10.1016/j.susc.2017.08.002

This is a PDF file of an unedited manuscript that has been accepted for publication. As a service to our customers we are providing this early version of the manuscript. The manuscript will undergo copyediting, typesetting, and review of the resulting proof before it is published in its final form. Please note that during the production process errors may be discovered which could affect the content, and all legal disclaimers that apply to the journal pertain. 


\section{Highlights}

- Graphene grown on $\mathrm{Ni}(111)$ surface alloyed with group IV atoms is studied by means of first principles methods.

- Alloyed surfaces reduce the interaction between graphene and Ni.

- New substrates are proposed for high quality graphene growth.

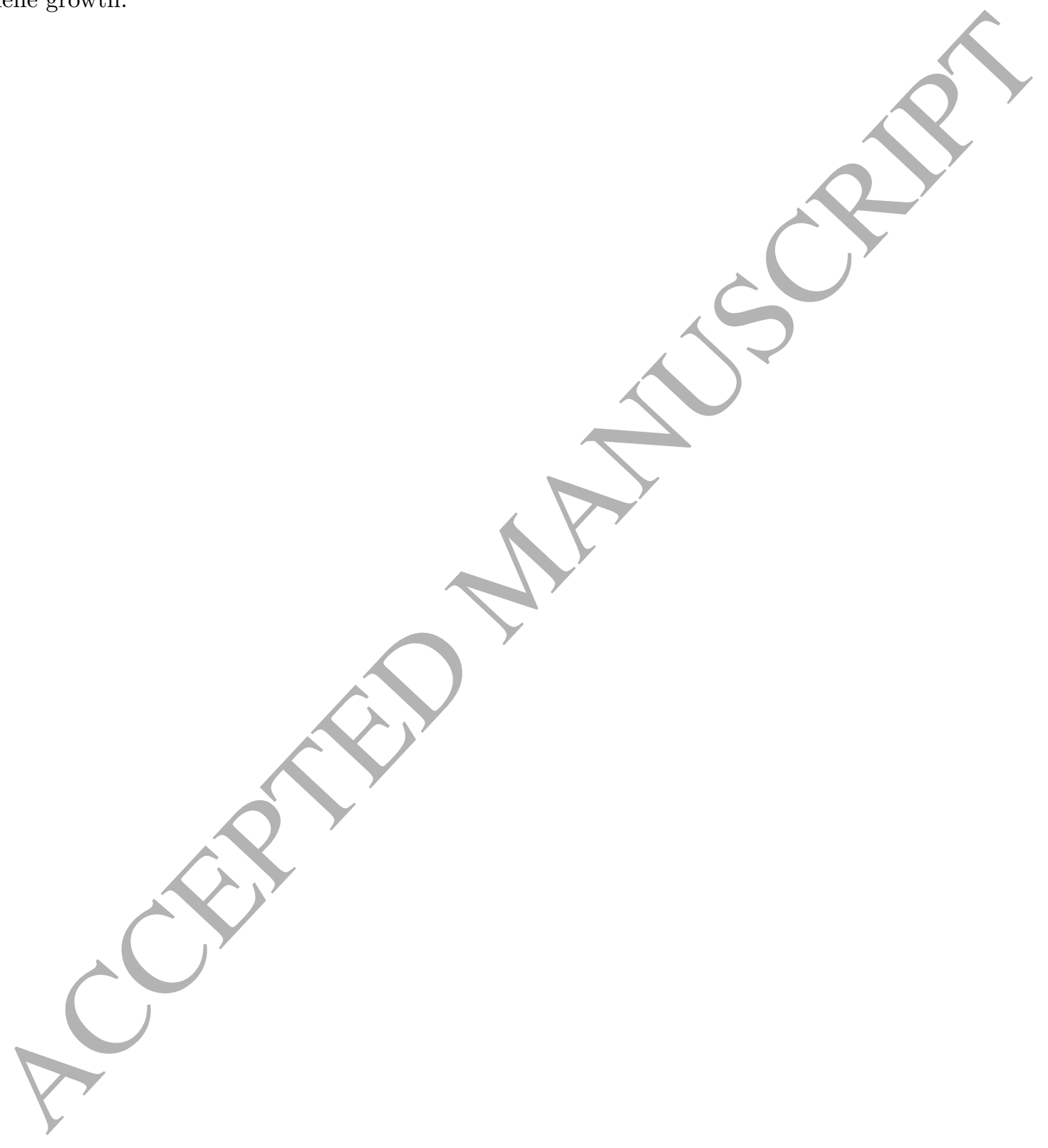



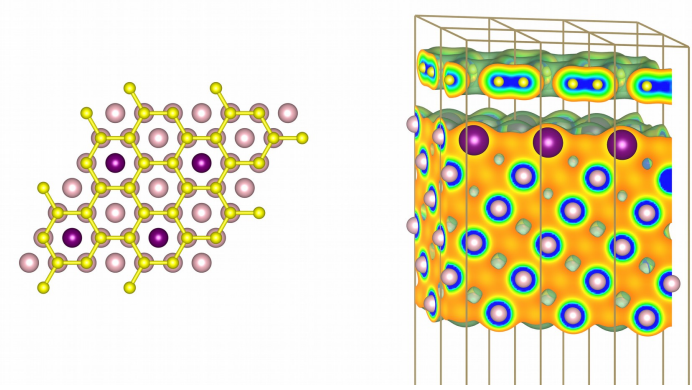

Top view (left) and charge distribution (right) of graphene on $\mathrm{GeNi}_{2}$ alloyed surface in the hcp-fcc staking. The detachment of graphene layer is evident.

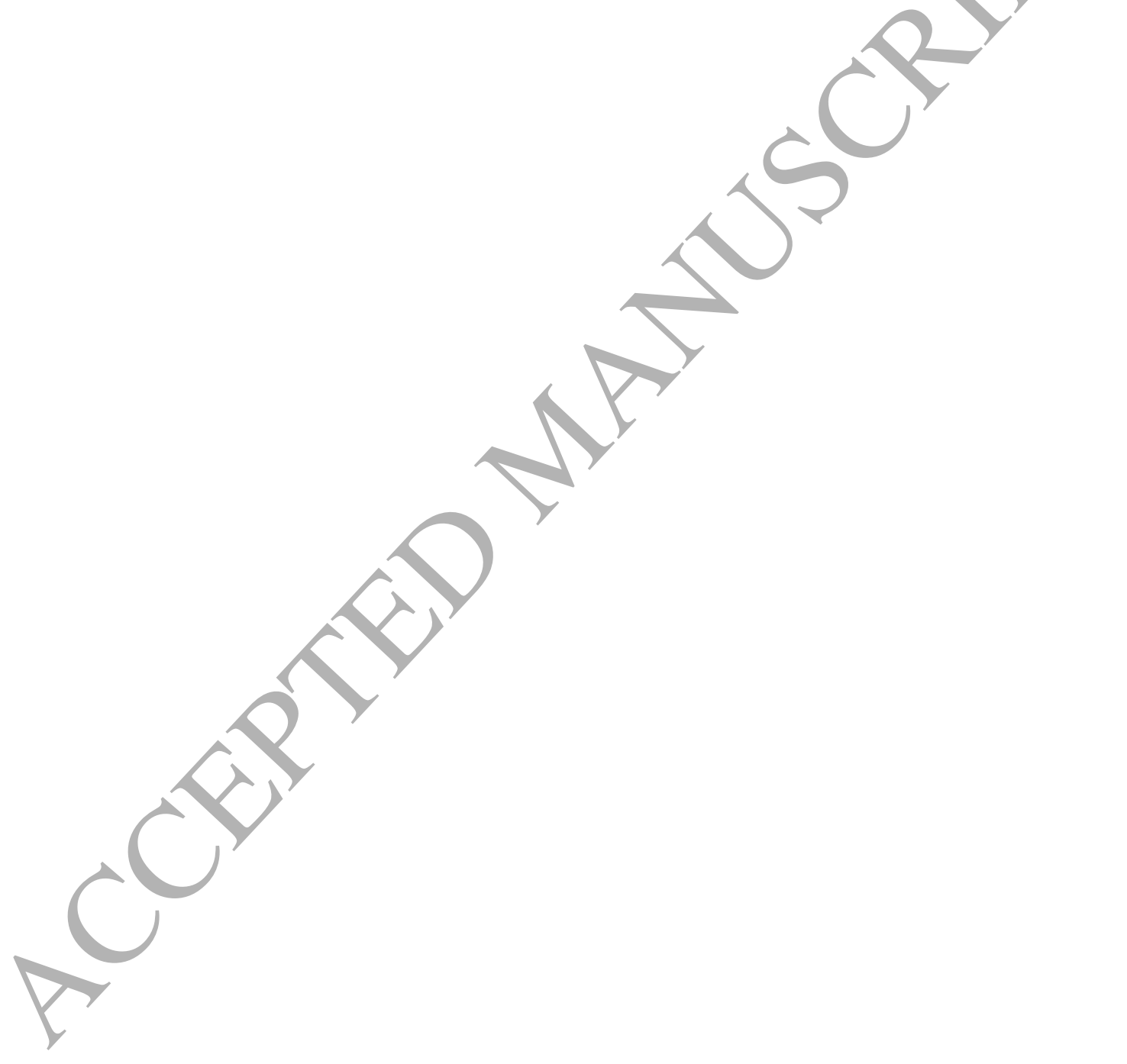




\title{
Alloyed surfaces: new substrates for graphene growth
}

\author{
C. Tresca ${ }^{\mathrm{a}, \mathrm{b}}$, N.I. Verbitskiy ${ }^{\mathrm{c}}$, A. Fedorov ${ }^{\mathrm{c}}$, A. Grüneis ${ }^{\mathrm{c}}$, G. Profeta ${ }^{\mathrm{a}, \mathrm{b}, *}$ \\ ${ }^{a}$ Department of Physical and Chemical Sciences, University of L'Aquila, Via Vetoio 10, I-67100, L'Aquila (Italy) \\ ${ }^{b}$ SPIN-CNR, University of L'Aquila, Via Vetoio 10, I-67100, L'Aquila (Italy) \\ ${ }^{c}$ II. Physikalisches Institut, Universität zu Köln, Zülpicher Straße 77, D-50937 Köln (Germany)
}

\begin{abstract}
We report a systematic ab-initio density functional theory investigation of $\mathrm{Ni}(111)$ surface alloyed with elements of group IV ( $\mathrm{Si}, \mathrm{Ge}$ and $\mathrm{Sn}$ ), demonstrating the possibility to use it to grow high quality graphene. Ni(111) surface represents an ideal substrate for graphene, due to its catalytic properties and perfect matching with the graphene lattice constant. However, Dirac bands of graphene growth on $\mathrm{Ni}(111)$ are completely destroyed due to the strong hybridization between carbon $p_{z}$ and Ni $d$ orbitals. Group IV atoms, namely $\mathrm{Si}$, Ge and $\mathrm{Sn}$, once deposited on Ni(111) surface, form an ordered alloyed surface with $\sqrt{3} \times \sqrt{3}-\mathrm{R} 30^{\circ}$ reconstruction. We demonstrate that, at variance with the pure $\mathrm{Ni}(111)$ surface, alloyed surfaces effectively decouple graphene from the substrate, resulting unstrained due to the nearly perfect lattice matching and preserves linear Dirac bands without the strong hybridization with $\mathrm{Ni} d$ states. The proposed surfaces can be prepared before graphene growth without resorting on post-growth processes which necessarily alter the electronic and structural properties of graphene.
\end{abstract}

Keywords: Graphene growth, electronic structure, alloyed surfaces, density functional theory PACS: 61.48.Gh, 68.65.Pq, 73.22.Pr, 68.35.bd

\section{Introduction}

The extraordinary properties of graphene $[1,2]$ mainly arise from its electronic features: linear dispersion of electronic bands (Dirac bands) originating from carbon $p_{z}$ orbitals around $K$ points of Brillouin Zone (BZ). Electrons have photons-like energy dispersion with zero rest mass showing extraordinary transport properties $[3]$.

In recent years, thanks to the improvement of large scale growth techniques, like chemical vapour deposition (CVD), it has been possible to obtain high quality graphene $[4,5,6]$ on different metal substrates allowing a deep investigation of the substrate's role in determining the structural and electronic properties of graphene.

The (111) surface of nickel $(\mathrm{Ni}(111))$ can be considered as an ideal substrate for graphene growth due to its catalytic properties and perfect matching with the graphene's lattice constant ( $a_{\text {graphene }}=2.46 \AA$ vs $\left.a_{N i}=2.49 \AA\right)$, in contrast with other metallic substrates. Unfortunately, Dirac bands of graphene growth on $\mathrm{Ni}(111)$ are completely destroyed due to the strong hybridization between carbon $p_{z}$ and $\mathrm{Ni} d$ orbitals and by the sublattice asymmetry induced by different position of the two carbons in the graphene unit cell. This behaviour has been recently questioned by means of ARPES measurements on graphene grown on $\mathrm{Ni}(111)$ and $\mathrm{Co}(0001)$ surfaces[7] that evidenced the presence of intact Dirac cones. However it was suggested that it is due to dynamic hybridization between $p_{z}$

\footnotetext{
*gianni.profeta@aquila.infn.it
}

and $d$ orbitals or to the stabilization of an high symmetry (hígh-energy) stacking (see below)[7].

In order to recover the linear dispersing Dirac bands, the intercalation with foreign atoms represents one of the most effective experimental techniques[8]: the deposition of adatoms on-top the graphene surface growth on Ni substrate followed by thermal treatment induces the intercalation of the adatoms in between the substrate and graphene, detaching it from the substrate. Different kind of atoms have been theoretically proposed and experimentally explored in the last few years $[8,9,10,11]$, but depending on the atoms, charge doping, structural disorder and gap opening can result after the intercalation process. For example, it is known that noble metals (like Au) are particular efficient to produce high quality graphene with neutrality point at zero[12], the same results have been recently obtained by Verbitskiy et al. $[13,14]$ by Ge intercalation in graphene growth on $\mathrm{Ni}(111)$; on the other hand, alkali metals can produce a splitting of the Dirac point[15] or returning an $n$-doped detached graphene like in the case of Cs intercalation[16, 17, 18].

Although intercalation is rather efficient, it represents a post-deposition process which is intrinsically not easily controllable: for example the annealing can induce defects and inhomogeneities in graphene, with subsequent variation of the stoichiometry, doping and structural properties.

The direct growth of graphene on a clean and ordered surface would be preferable, possibly reducing postdeposition treatments and uncontrolled defect formations. 
In the last few years it was demonstrated that Ni-based alloys can be an effective and promising substrate for large scale graphene growth[19, 20, 21]. In this paper we propose $\mathrm{Ni}(111)$ surface, alloyed with group IV atoms, as alternative and promising substrates for free-standing-like graphene growth.

Transition metal surfaces were already reported to form ordered two-dimensional phases when alloyed with different atoms. Overbury and $\mathrm{Ku}[22]$ in 1992 found the formation of a stable two dimensional phase of Sn deposited on $\mathrm{Cu}(111), \mathrm{Ni}(111)$, and $\mathrm{Pt}(111)$ surfaces. The authors, by means of LEED measurements, observed a surface reconstruction with $(\sqrt{3} \times \sqrt{3})-\mathrm{R} 30^{\circ}(\sqrt{3})$ symmetry with respect to the underlying $\mathrm{Ni}(111)(1 \times 1)$ phase, and the effective formation of the surface alloy, with Sn substituting surface $\mathrm{Ni}$ atom. The subject was recently re-examined by Rahman et al.[23], which have demonstrated that the same ordered structure can be obtained depositing silicon and germanium on-top the $\mathrm{Ni}(111)$ surface followed by thermal annealing at $650 \mathrm{~K}$. Structural analysis revealed a $\mathrm{XNi}_{2}(\mathrm{X}=\mathrm{Si}, \mathrm{Ge})$ stoichiometry of the surface, in agreement with XPS[13]. Interestingly, the alloyed phases are rather stable: for example the $\sqrt{3}$ reconstruction of $\mathrm{SnNi}_{2}$ is still present up to temperature of about $1000 \mathrm{~K}[22]$ (850K for $\left.\mathrm{Ge}(\mathrm{Si}) \mathrm{Ni}_{2}[23]\right)$.

Based on these experimental evidences, by means of first-principles calculations, we predict the structural and electronic properties of graphene growth on alloyed $\mathrm{Ni}(111)$ surfaces demonstrating that, $(i)$ good lattice matching reduces strain effects, (ii) group IV atoms avoid the strong hybridization between $\mathrm{Ni}-d$ states and graphene, de - facto decoupling graphene from the substrate and (iii) graphene results with Dirac point close to zero neutrality level. In addition, being derived from nickel, the alloyed substrates should have the required catalytic properties to allow the direct growth of graphene, without any post-deposition process.

\section{Computational details}

The calculations were performed within first-principles Density Functional Theory using the Vienna Ab-Initio Simulation Package (VASP) [24, 25].

We used Projected Augmented-Wave (PAW) pseudopotentials[26] and local density approximation (LDA) for the exchange correlation energy with an energy cutoff of $500 \mathrm{eV}$. The surfaces were simulated within a supercell approach considering six Ni layers with a $\sqrt{3}$ reconstruction (following the experimental results, see below) and $24 \AA$ of vacuum. Graphene was adsorbed on the topmost $\mathrm{Ni}$ surface layer. Integration of charge density over the two dimensional Brillouin zone (BZ) was performed using an uniform $6 \times 6$ Monkhorst and Pack grid[27] with a Gaussian smearing parameter $\sigma=0.1 \mathrm{eV}$.

Both ferromagnetic (FM) and non-magnetic (NM) solutions were considered in the calculations. Structural optimization was allowed for all the atoms but the first four
Ni layers (bulk-like) which are fixed to their ideal Ni bulk positions.

\section{Results and discussion}

The description of graphene stacking on $\mathrm{Ni}(111)$ already represents per se a challenging problem from a computational point of view, due to difficulties to take into account dispersive forces and many-body effects beyond the local density approximation[28]. However, it was demonstrated that LDA accounts for many important structural and electronic properties of graphene on $\mathrm{Ni}(111)$ surface finding a quantitative agreement with experiments[29]. The total energy for different stacking of graphene on $\mathrm{Ni}$ surface (see Fig.1), using LDA, are summarized in Tab.1.

In agreement with previous first-principles calculations[30, 31], we predict the bridge-top as the ground state stacking (for both NM and FM phases, see Tab.1). We note that bridge-fcc and bridge-hcp configurations (Fig.1(a) and Fig.1(b), respectively) are not stable and relax into the top-fcc or top-hcp upon structural optimization which are, indeed, close in energy with the ground state. Inclusion of spin-polarization does not alter the qualitative results. The analysis of the structural properties reveals that graphene stands essentially at two different height from the substrate, depending on the stacking: about $2 \AA$ (for bridge-top, top-fcc and top-hcp) and higher than $3 \AA$ (for hcp-fcc). This aspect is crucial in predicting graphene's electronic properties.

\begin{tabular}{|c|c|c|}
\cline { 2 - 3 } \multicolumn{1}{c|}{} & $d$ & $\Delta E$ \\
\hline Bridge-top & $1.93(1.95)$ & $0.00(0.00)$ \\
\hline Hcp-fcc & $3.21(3.31)$ & $1.28(1.06)$ \\
\hline Top-fcc & $1.99(2.02)$ & $0.13(0.09)$ \\
\hline Top-hcp & $2.00(2.02)$ & $0.26(0.18)$ \\
\hline
\end{tabular}

Table 1: Structural and energetic properties of graphene on $\mathrm{Ni}$ substrate in different stacking configurations for NM (FM) phase. We report the distance between graphene and substrate surface $(d(\AA))$ and the total energy with respect the ground state $(\Delta E(\mathrm{eV}))$

The electronic band structure of the graphene/Ni(111) surface (in the ground state phase), reported in Fig.2 (top panel), confirms that the $\mathrm{C} p_{z}$ and $\mathrm{Ni} d$ orbitals are strongly hybridized destroying the graphene Dirac cones. On the contrary, the band structure of graphene in the hcp-fcc phase (Fig.2, lower panel), resembles that of the quasi free-standing system, thanks to the large distance between graphene and Ni surface (see Tab.1). However, this last phase is not thermodynamically stable.

As discussed in the introduction, while the intercalation by foreign atoms can recover a quasi free standing 


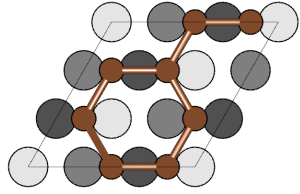

(a) Bridge-fcc

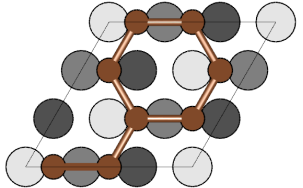

(b) Bridge-hcp

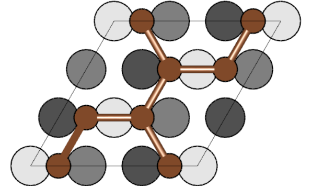

(c) Bridge-top

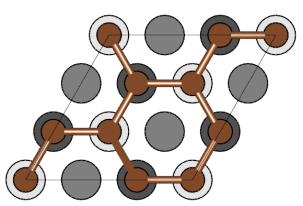

(e) Top-fcc

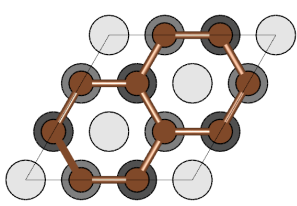

(d) Hcp-fcc (f) Top-hcp

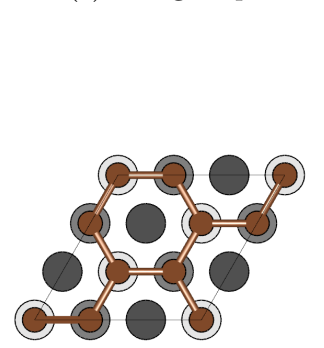

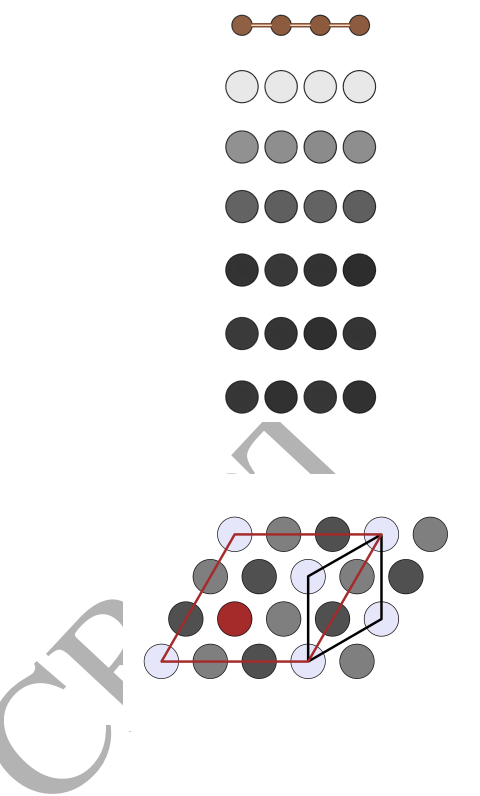

Figure 1: Considered stacking of graphene (brown atoms) on $\mathrm{Ni}(111)$ surface. Different Ni layers are reported in gray scale from light (topmost layer) to dark (inner layer) (see the lateral view on the right-top panel). In the right-bottom panel we report the $(\sqrt{3} \times \sqrt{3})$-R30 (red unit cell) structure of alloyed surface with IV-group adatom (red) substituting a surface $\mathrm{Ni}$ atom (the $1 \times 1$ unit cell is also reported in black for reference).

graphene, here we propose a different strategy: stabilize high energy stacking phases of graphene/Ni(111) systems tuning the chemical composition of the substrate. The possibility to obtain a surface alloying with group IV atoms on $\mathrm{Ni}(111)$ surface represents a possible strategy we want to explore.

As experimentally demonstrated the $\sqrt{3}$ phase is formed substituting $1 / 3$ of monolayer of $\mathrm{Ni}$ with of group IV atom, with a final stoichiometry of $\mathrm{XNi}_{2}(\mathrm{X}=\mathrm{Si}, \mathrm{Ge}$ and $\mathrm{Sn})$ (see Fig.1).

\begin{tabular}{|l|c|c|c|}
\cline { 2 - 4 } \multicolumn{1}{c|}{} & \multicolumn{1}{c|}{$\mathrm{SiNi}_{2}$} & $\mathrm{GeNi}_{2}$ & $\mathrm{SnNi}_{2}$ \\
\hline Bridge-top & $2.07 / 0.03$ & $2.15 / 0.12$ & $2.15 / 0.88$ \\
\hline Hcp-fcc & $3.44 / 0.16$ & $3.53 / 0.00$ & $3.88 / 0.03$ \\
\hline Top-fcc & $2.14 / 0.00$ & $2.14 / 0.08$ & $3.77 / 0.00$ \\
\hline Top-hcp & $2.22 / 0.04$ & $2.30 / 0.11$ & $3.78 / 0.00$ \\
\hline
\end{tabular}

Table 2: Graphene on $\mathrm{XNi}_{2}$ substrate in different stacking configurations. We report the distance between graphene and substrate $(d(\AA))$ and the energy with respect the ground state $(\Delta E(e V))$ for each compounds $(d / \Delta E)$

The structural and energetic properties of graphene on $\mathrm{XNi}_{2}$ surface are summarized in Tab.2. As in the case of pristine $\mathrm{Ni}(111)$ substrate, the conclusions of the FM calculations qualitatively agree with the NM ones and are not reported. Already for the first substrate considered, $\mathrm{SiNi}_{2}$, we find an important difference with respect to pure $\mathrm{Ni}$ surface: the most stable configuration for graphene is the top-fec. However, although the graphene-Ni(111) distance increases after Si alloying, graphene still remains quite close to substrate, mainly due to the small Si ionic radius, still indicating a strong binding.

As a general trend (common to all the considered stacking), we observe that the Si atom is pulled down into the $\mathrm{Ni}$ surface, mainly due to the smaller ionic radius of $\mathrm{Si}$ with respect to $\mathrm{Ni}$. Increasing the effective ionic size of the substitutional atom, thus considering $\mathrm{GeNi}_{2}$, the opposite trend is observed: Ge atoms protrude from the surface and the most stable configuration results the hcp-fcc with an effective distance of $3.53 \AA$ (see Tab.2).

The $\mathrm{SnNi}_{2}$ surface follows the same trend: $\mathrm{Sn}$ stands out from the surface for all the considered configurations (except in the case of bridge-top, which results stabilized by the strength of chemical bond between graphene and Ni top atoms, despite an high formation energy). We find the top-fcc structure as the ground state phase, nearly degenerate with the top-hcp stacking, resulting in a very large graphene-Ni(111) distance (3.77 $\AA$, see Tab.2).

The stabilization of high distance stacking can be ascribed to a structural feature. The electronic properties of group IV atoms do not depend on the graphene's stacking: from the density of states projected on surface atoms (not shown), we observe that, for all the considered systems, group IV atoms slightly dope Ni surface, irrespective of the stacking configuration. Thus, it is the larger ionic radius of the dopants with respect to $\mathrm{Ni}$ atom, that causes the protrusion from the surface and the subsequent increase of the graphene distance.

Based on the structural properties of the three different 


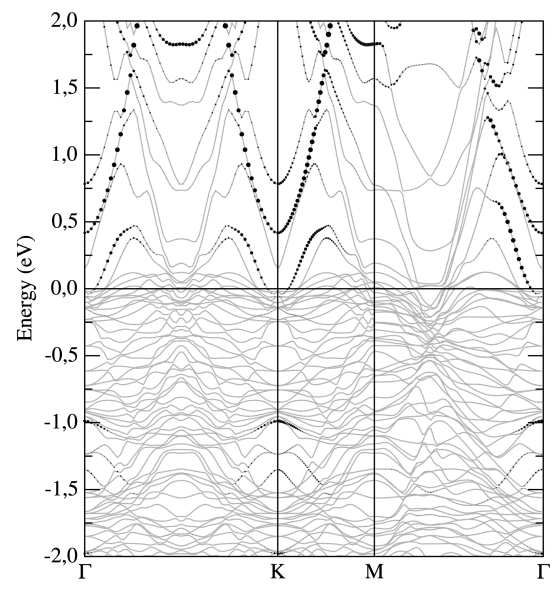

(a) Top-fcc on $\mathrm{SiNi}_{2}$

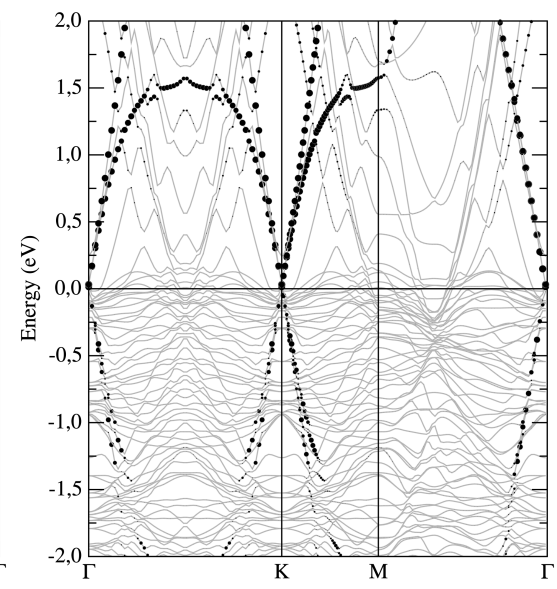

(b) Hcp-fcc on $\mathrm{GeNi}_{2}$

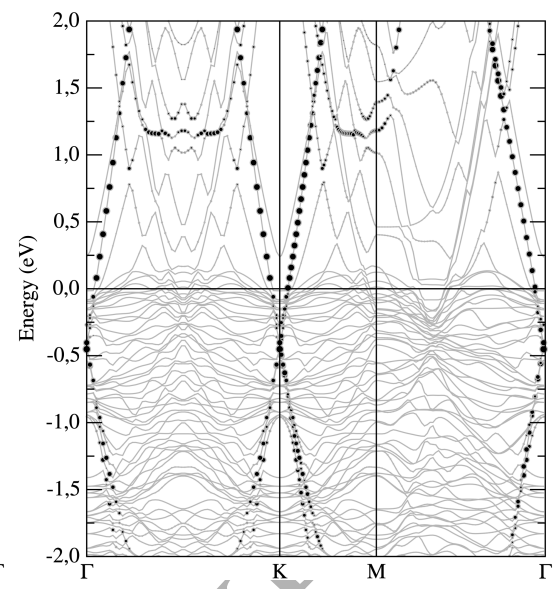

(c) Top-fcc on $\mathrm{SnNi}_{2}$

Figure 3: Electronic dispersion for graphene on $\mathrm{XNi}_{2}(\mathrm{X}=\mathrm{Si}$, Ge and $\mathrm{Sn})$ in the $(\sqrt{3} \times \sqrt{3})$ - $\mathrm{R} 30^{\circ}$ ground state geometry (indicated at the bottom of the figure). The size of the circles for each eigenvalue is proportional to the weight of the corresponding wavefunction on the carbon sites.

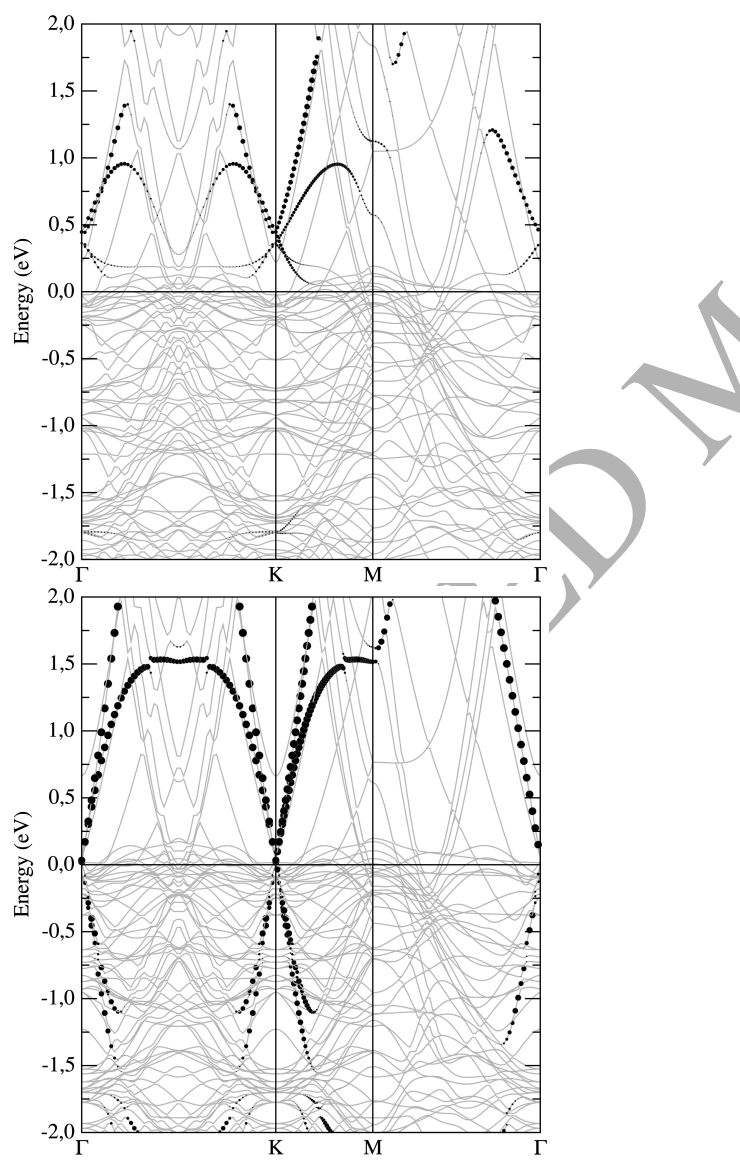

Figure 2: Electronic dispersion for graphene on $\mathrm{Ni}(111)$ in the $(\sqrt{3} \times \sqrt{3})-\mathrm{R} 30^{\circ}$ ground state geometry (upper panel) and hcp-fcc phase (lower panel). The size of the circles for each eigenvalue is proportional to the weight of the corresponding wavefunction on the carbon sites. substrates, we can infer that $\mathrm{GeNi}_{2}$ and $\mathrm{SnNi}_{2}$ substrates can be suitable to growth a quasi-free-standing graphene. In order to confirm the details of graphene electronic properties on the three different substrates, we calculated the band structure projected on the graphene atoms (Fig.3). We observe that Dirac bands does not exists in graphene grown on $\mathrm{SiNi}_{2}$ due to strong hybridization with substrate, similar to pure $\mathrm{Ni}$, while $\mathrm{GeNi}_{2}$ and $\mathrm{SnNi}_{2}$ substrates allow to recover linear Dirac bands at the Fermi energy. In addition, in the $\mathrm{GeNi}_{2}$ case, the graphene's neutrality point is close to zero $(+0.02 \mathrm{eV})$. $\mathrm{SnNi}_{2}$ substrate induces an electron doping, with Dirac point at about $-0.43 \mathrm{eV}$ below Fermi energy in both the degenerate stacking.

\section{Conclusion}

In present work, we studied the structural and electronic properties of graphene on $\mathrm{XNi}_{2}$ alloyed surfaces (with $\mathrm{X}=\mathrm{Si}, \mathrm{Ge}, \mathrm{Sn}$ ). We have demonstrated that the substrates determine different stacking of graphene due to the different ionic radius of substitutional atoms. We studied the possibility to growth graphene on alloyed surfaces and we found several indications to consider $\mathrm{GeNi}_{2}$ and $\mathrm{SnNi}_{2}$ as suitable substrates to stabilize quasi-freestanding graphene: $i$ ) the rippling of the surface induced by the alloying guarantees the structural decoupling of graphene from the substrate with distances higher than $3 \AA$, $i i)$ the electronic dispersion preserve the linear Dirac bands near the Fermi energy, iii) graphene on $\mathrm{GeNi}_{2}$ substrate results neutral (while when growth on $\mathrm{SnNi}_{2}$ it is $n$-doped), iv) Ni-rich surfaces should guarantee comparable catalytic properties like pure $\mathrm{Ni}(111)$ surface, $v$ ) postgrowth processes can be avoided. We believe that the proposed surfaces can represent an important alternative for high quality quasi-free-standing graphene growth and call for further experimental confirmation. 


\section{Acknowledgments}

We acknowledge the CINECA award under the ISCRA initiative, for access to high performance computing resources and support.

\section{References}

[1] K. S. Novoselov, A. K. Geim, S. V. Morozov, D. Jiang, Y. Zhang, S. V. Dubonos, I. V. Grigorieva, A. A. Firsov, Electric field effect in atomically thin carbon films, Science 306 (5696) (2004) 666-669. arXiv:http://science.sciencemag.org/content/306/5696/666.full.pdf, doi:10.1126/science.1102896.

URL http://science.sciencemag.org/content/306/5696/666

[2] K. S. Novoselov, D. Jiang, F. Schedin, T. J. Booth, V. V. Khotkevich, S. V. Morozov, A. K. Geim, Two-dimensional atomic crystals, Proceedings of the National Academy of Sciences of the United States of America 102 (30) (2005) 10451-10453. arXiv:http://www.pnas.org/content/102/30/10451.full.pdf, doi:10.1073/pnas.0502848102.

URL http://www . pnas.org/content/102/30/10451. abstract

[3] A. K. Geim, K. S. Novoselov, The rise of graphene, Nat Mater 6 (2007) 183-191.

[4] X. Li, W. Cai, J. An, S. Kim, J. Nah, D. Yang, R. Piner, A. Velamakanni, I. Jung, E. Tutuc, S. K. Banerjee, L. Colombo, R. S. Ruoff, Large-area synthesis of high-quality and uniform graphene films on copper foils, Science 324 (5932) (2009) 1312-1314. arXiv:http://science.sciencemag.org/content/324/5932/1312.full.pdf doi:10.1126/science.1171245.

URL http://science.sciencemag.org/content/324/5932/1312

[5] J.-H. Lee, E. K. Lee, W.-J. Joo, Y. Jang, B.-S. Kim, J. Y. Lim, S.-H. Choi, S. J. Ahn, J. R. Ahn, M.-H. Park, C.-W. Yang, B. L. Choi, S.-W. Hwang, D. Whang, Wafer-scale growth of single-crystal monolayer graphene on reusable hydrogenterminated germanium, Science 344 (6181) (2014) 286-289. arXiv:http://science.sciencemag.org/content/344/6181/286.full.pdf, doi:10.1126/science.1252268.

URL http://science.sciencemag.org/content/344/6181/286

[6] Z.-D. Liu, Z.-Y. Yin, Z.-H. Du, Y. Yang, M.-M. Zhu, L.-H. Xie, W. Huang, Low temperature growth of graphene on cu-ni alloy nanofibers for stable, flexible electrodes, Nanoscale 6 (10) (2014) 5110. doi:10.1039/c3nr06246e.

URL https://doi.org/10.1039/c3nr06246e

[7] A. Varykhalov, D. Marchenko, J. Sánchez-Barriga, M. R. Scholz, B. Verberck, B. Trauzettel, T. O. Wehling, C. Carbone, O. Rader, Intact dirac cones at broken sublattice symmetry: Photoemission study of graphene on ni and co, Phys. Rev. X 2 (2012) 041017. doi:10.1103/PhysRevX.2.041017.

URL https://link.aps.org/doi/10.1103/PhysRevX.2.041017

[8] E. V. Rut'kov, N. R. Gall, Penetration (intercalation) of copper atoms under a graphene layer on iridium (111), Semiconductors 43 (10) (2009) 1255-1258. doi:10.1134/S1063782609100017. URL http://dx.doi.org/10.1134/S1063782609100017

[9] C. Virojanadara, S. Watcharinyanon, A. A. Zakharov, L. I. Johansson, Epitaxial graphene on $6 h-\mathrm{SiC}$ and li intercalation, Phys. Rev. B 82 (2010) 205402. doi:10.1103/PhysRevB.82.205402.

URL http://link.aps.org/doi/10.1103/PhysRevB.82.205402

[10] S. Watcharinyanon, C. Virojanadara, L. Johansson, Rb and Cs deposition on epitaxial graphene grown on 6hsic(0001), Surface Science 605 (2122) (2011) 1918 - 1922. doi:http://dx.doi.org/10.1016/j.susc.2011.07.007.

URL http://www.sciencedirect.com/science/article/pii/S003

[11] F. Bisti, G. Profeta, H. Vita, M. Donarelli, F. Perrozzi, P. M. Sheverdyaeva, P. Moras, K. Horn, L. Ottaviano, Electronic and geometric structure of graphene/sic(0001) decoupled by lithium intercalation, Phys. Rev. B 91 (2015) 245411.
doi:10.1103/PhysRevB.91.245411.

URL https://link.aps.org/doi/10.1103/PhysRevB.91.245411

[12] E. Voloshina, Y. Dedkov, Graphene on metallic surfaces: problems and perspectives, Phys. Chem. Chem. Phys. 14 (2012) 13502-13514. doi:10.1039/C2CP42171B.

URL http://dx.doi.org/10.1039/C2CP42171B

[13] N. I. Verbitskiy, A. V. Fedorov, G. Profeta, A. Stroppa, L. Petaccia, B. Senkovskiy, A. Nefedov, C. Wöll, D. Y. Usachov, D. V. Vyalikh, L. V. Yashina, A. A. Eliseev, T. Pichler, A. Grüneis, Atomically precise semiconductor-graphene and hbn interfaces by ge intercalation, Scientific Reports 5 (2015) 17700 .

[14] N. I. Verbitskiy, A. V. Fedorov, C. Tresca, G. Profeta, L. Petaccia, B. V. Senkovskiy, D. Y. Usachoy, D. V. Vyalikh, L. V. Yashina, A. A. Eliseev, T. Pichler, A. Grneis, Environmental control of electronphonon coupling in barium doped graphene, 2D Materials 3 (4) (2016) 045003. URL http://stacks.iop.org/2053-1583/3/i=4/a=045003

[15] A. Grüneis, D. V. Vyalikh, Tunable hybridization between electronic states of graphene and a metal surface, Phys. Rev. B 77 (2008) 193401. doi:10.1103/PhysRevB.77.193401.

URL http://link.aps.org/doi/10.1103/PhysRevB.77.193401

[16] N. Ligato, A. Cupolillo, L Caputi, Study of the intercalation of graphene on ni(111) with cs atoms: Towards the quasi-free graphene, Thin Solid Films 543 (2013) 59 - 62, international Conference NanoSEA (NANOstructures SElf Assembly) 2012. doi:http://dx.doi.org/10.1016/j.tsf.2013.02.121.

URL http://www.sciencedirect.com/science/article/pii/S004060901:

[17] A. Cupolillo, N. Ligato, L. S. Caputi, Plasmon dispersion in quasi-freestanding graphene on ni(111), Applied Physics Letters 102 (11) (2013) 111609. arXiv:http://dx.doi.org/10.1063/1.4798331, doi:10.1063/1.4798331.

URL http://dx.doi.org/10.1063/1.4798331

M. Alattas, U. Schwingenschlögl, Quasi-freestanding graphene on ni(111) by cs intercalation, Scientific Reports 6 (1). doi:10.1038/srep26753.

URL https://doi.org/10.1038/srep26753

[19] T. Wu, X. Zhang, Q. Yuan, J. Xue, G. Lu, Z. Liu, H. Wang, H. Wang, F. Ding, Q. Yu, X. Xie, M. Jiang, Fast growth of inch-sized single-crystalline graphene from a controlled single nucleus on cu-ni alloys, Nature Materials 15 (1) (2015) 43-47. doi:10.1038/nmat4477.

URL https://doi.org/10.1038/nmat4477

[20] Y. Feng, X. Yao, Z. Hu, J.-J. Xu, L. Zhang, Passivating a transition-metal surface for more uniform growth of graphene: Effect of au alloying on ni(111), Phys. Rev. B 87 (2013) 195421. doi:10.1103/PhysRevB.87.195421.

URL https://link.aps.org/doi/10.1103/PhysRevB.87.195421

[21] A. Politano, G. Chiarello, Graphene on pt 3 ni(111): a suitable platform for tunable charge doping, electronphonon coupling and plasmonic excitations, 2D Materials 4 (3) (2017) 035003. URL http://stacks . iop.org/2053-1583/4/i=3/a=035003

[22] S. H. Overbury, Y.-s. Ku, Formation of stable, two-dimensional alloy-surface phases: $\mathrm{Sn}$ on $\mathrm{Cu}(111), \mathrm{Ni}(111)$, and $\mathrm{Pt}(111)$, Phys. Rev. B 46 (1992) 7868-7872.

[23] M. S. Rahman, T. Nakagawa, S. Mizuno, Structure determination of the ordered phase of $\mathrm{Ni}_{2} \mathrm{Si}$ and $\mathrm{Ni}_{2} \mathrm{Ge}$ surface alloys on $\mathrm{Ni}(111)$ via low-energy electron diffraction, Surface Science 642 (2015) 1 - 5. doi:http://dx.doi.org/10.1016/j.susc.2015.07.024. URL http://www. sciencedirect.com/science/article/pii/S003960281!

[24] G. Kresse, J. Furthmüller, Efficient iterative schemes for ab-initio total energy calculations using a planewave basis set, Phys. Rev. B 54 (1996) 11169-11186. doi:10.1103/PhysRevB.54.11169. URL http://link.aps.org/doi/10.1103/PhysRevB.54.11169

[25] G. Kresse, J. Furthmüller, Efficiency of ab-initio total energy $11.0122_{949} 40$ for metals and semiconductors using a plane-wave basis set, Computational Materials Science 6 (1) (1996) 15 50. doi:http://dx.doi.org/10.1016/0927-0256(96)00008-0. URL http://www.sciencedirect.com/science/article/pii/09270256961 
[26] P. E. Blöchl, Projector augmented-wave method, Phys. Rev. B 50 (1994) 17953-17979. doi:10.1103/PhysRevB.50.17953.

URL http://link.aps.org/doi/10.1103/PhysRevB.50.17953

[27] H. J. Monkhorst, J. D. Pack, Special points for Brillouinzone integrations, Phys. Rev. B 13 (1976) 5188-5192. doi:10.1103/PhysRevB.13.5188.

URL http://link.aps.org/doi/10.1103/PhysRevB.13.5188

[28] F. Mittendorfer, A. Garhofer, J. Redinger, J. Klimeš, J. Harl, G. Kresse, Graphene on ni(111): Strong interaction and weak adsorption, Phys. Rev. B 84 (2011) 201401. doi:10.1103/PhysRevB.84.201401.

URL http://link.aps.org/doi/10.1103/PhysRevB.84.201401

[29] F. Bianchini, L. L. Patera, M. Peressi, C. Africh, G. Comelli, Atomic scale identification of coexisting graphene structures on $\mathrm{Ni}(111)$, The Journal of Physical Chemistry Letters 5 (3) (2014) 467-473, pMID: 26276594. doi:10.1021/jz402609d.

[30] M. Fuentes-Cabrera, M. I. Baskes, A. V. Melechko, M. L. Simpson, Bridge structure for the graphene/Ni(111) system: A first principles study, Phys. Rev. B 77 (2008) 035405. doi:10.1103/PhysRevB.77.035405.

URL http://link.aps.org/doi/10.1103/PhysRevB.77.035405

[31] W. Zhao, S. M. Kozlov, O. Höfert, K. Gotterbarm, M. P. A. Lorenz, F. Viñes, C. Papp, A. Görling, H.-P. Steinrück, Graphene on Ni(111): Coexistence of different surface structures, The Journal of Physical Chemistry Letters 2 (7) (2011) 759-764. arXiv:http://dx.doi.org/10.1021/jz200043p, doi:10.1021/jz200043p.

URL http://dx.doi.org/10.1021/jz200043p 